Research Article

\title{
Distance-Based Polynomials and Topological Indices for Hierarchical Hypercube Networks
}

\author{
Tingmei Gao ${ }^{1}{ }^{1}$ and Iftikhar Ahmed ${ }^{2}$ \\ ${ }^{1}$ School of Mathematical and Computer Science, Shaanxi University of Technology, Hanzhong 723000, China \\ ${ }^{2}$ Department of Mathematics, Riphah International University, Lahore Campus, Lahore 54000, Pakistan
}

Correspondence should be addressed to Tingmei Gao; dghnn021@163.com

Received 19 July 2021; Revised 2 August 2021; Accepted 16 August 2021; Published 11 September 2021

Academic Editor: Muhammad Kamran Siddiqui

Copyright (C) 2021 Tingmei Gao and Iftikhar Ahmed. This is an open access article distributed under the Creative Commons Attribution License, which permits unrestricted use, distribution, and reproduction in any medium, provided the original work is properly cited.

\begin{abstract}
Topological indices are the numbers associated with the graphs of chemical compounds/networks that help us to understand their properties. The aim of this paper is to compute topological indices for the hierarchical hypercube networks. We computed Hosoya polynomials, Harary polynomials, Wiener index, modified Wiener index, hyper-Wiener index, Harary index, generalized Harary index, and multiplicative Wiener index for hierarchical hypercube networks. Our results can help to understand topology of hierarchical hypercube networks and are useful to enhance the ability of these networks. Our results can also be used to solve integral equations.
\end{abstract}

\section{Introduction}

The field of mathematics which deals with the problems of chemistry mathematically is mathematical chemistry. The topology of chemical structures, for example, topological labels or indices, is investigated in chemical graph theory. Actually, topological indices are real numbers associated with graph of chemical compounds and are useful in quantitative structure-property relationships and quantitative structure-activity relationships. Topological indices predict some important properties of chemical structures even without using lab, for example, boiling point, viscosity, radius of gyration, and so on [1-3] can be obtained from the indices.

Just like topological indices, polynomials also have significant applications in chemistry, for example, Hosoya polynomial [4] plays a vital role in calculation of distancebased topological indices. Like Hosoya polynomial, M-polynomial [5] plays the same role in calculation of many degree-based TIs [6-12].

Wiener defined the first topological index when he was examining boiling point of paraffins [13]. Consequently, Wiener set up the skeleton of topological indices [14-18].
This paper concerns with the topological indices of hierarchical hypercube networks. Interconnection networks have a pivotal role in the execution of parallel systems. This paper studies the interconnection topology that is called the hierarchical hypercube (HHC) [19]. This topology is suitable for extensively parallel systems with many number of processors. An appealing property of this network is the low number of connections per processor, which enhances the VLSI design and fabrication of the system $[20,21]$. Other alluring features include symmetry and logarithmic diameter, which imply easy and fast algorithms for communication [22]. Moreover, the HHC is scalable, that is, it can embed HHCs of lower dimensions. Malluhi and Bayoumi [21] introduced the hierarchical hypercube network of order $n$ ( $n-\mathrm{HHC})$. The structure of an $n-\mathrm{HHC}$ consists of three levels of hierarchy. At the lowest level of hierarchy, there is a pool of $2 n$ nodes. These nodes are grouped into clusters of $2 m$ nodes each, and the nodes in each cluster are interconnected to form an $m$-cube called the Son-cube or the $\mathrm{S}$-cube. The set of the S-cubes constitutes the second level of hierarchy [23].

Being a hierarchical structure, the HHC bears the advantages usually gained by hierarchy [24]. In general, 
hierarchy is a useful means for modular design. In addition, hierarchical structures are capable of exploiting the locality of reference (communication), and they are fault tolerant [25]. Other attractive properties of the HHC structure are logarithmic diameter and a topology inherited from, and closely related to, the hypercube topology. The former property implies fast communication, and the latter implies easy mapping of operations from HC to HHC. The HHC can emulate the hypercube for a large class of problems (divide and conquer), without a significant increase in processing time. The HHC can embed rings and HHCs of lower dimension. In addition, the HHC embeds the cube connected cycle (CCC) [26-28]. As a result, the performance of HHC is in the worst case equivalent to the performance of the CCC [29-34]. The number of vertices and edges in $(\mathrm{HHC}-1)$ is $16 a+16$ and $24 a+20$, respectively, where $a$ is a natural number. The number of vertices and edges in $(\mathrm{HHC}-2)$ is $16 a+16$ and $32 a+28$, respectively. $(\mathrm{HHC}-1)$ and $(\mathrm{HHC}-2)$ are shown in Figures 1 and 2, respectively.

In this paper, we computed Hosoya polynomials, Harary polynomials, Wiener index, modified Wiener index, hyperWiener index, Harary index, generalized Harary index, and multiplicative Wiener index for hierarchical hypercube networks.

\section{Preliminaries}

In this section, we give the definitions and known results that are used in proving main results of this paper. A graph $G$ is simple if it has no loop or multiple edges and is connected if there is a path between every two vertices of it. The distance between any two vertices $u$ and $v$ is denoted by $d(u, v)$ and is the length of the shortest path between $u$ and $v$. The diameter of a graph is the maximum distance between any two vertices of $G$. The notions that are used in this paper but not defined can be found in $[35,36]$.

Definition 1 (Hosoya polynomial [16]).

For a simple connected graph $G$, the Hosoya polynomial is defined as

$$
H(G, x)=\frac{1}{2} \sum_{y \in V(G)} \sum_{z \in V(G)} x^{d(y, z)},
$$

where $d(y, z)$ represents the distance between the vertices $y$ and $z$.

Definition 2 (Wiener index [37]). The Wiener index for a simple connected graph $G$ is denoted by $W(G)$ and is defined as the sum of distances between all pairs of vertices in $G$, i.e.,

$$
W(G)=\frac{1}{2} \sum_{y \in V(G)} \sum_{z \in V(G)} d(y, z) .
$$

It can be observed that the Wiener index is the first-order derivative of the Hosoya polynomial at $x=1$.

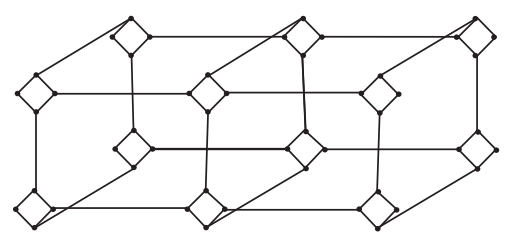

FIgURE 1: The hierarchical hypercube network $(\mathrm{HHC}-1)_{2 \times 2}$.

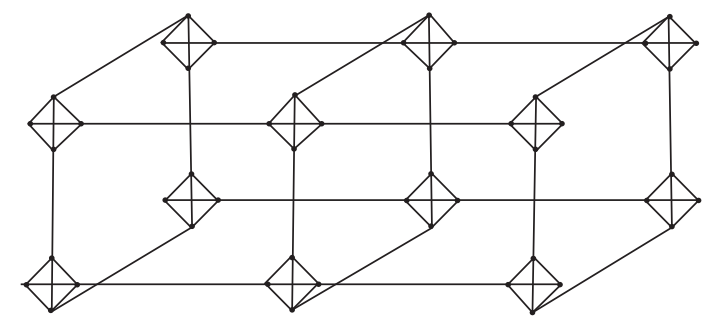

FIgURe 2: The hierarchical hypercube network $(\mathrm{HHC}-2)_{2 \times 2}$.

$$
W(G)=\left.\frac{\mathrm{d} H(G)}{\mathrm{d} x}\right|_{x=1}
$$

Definition 3 (modified Wiener index). For a simple connected graph $G$, the modified Wiener index is denoted by $W_{\lambda}(G)$ and is defined as

$$
W_{(\lambda G)}=\frac{1}{2} \sum_{y \in V(G)} \sum_{z \in V(G)} d(y, z)^{\lambda},
$$

where $\lambda$ is any positive integer.

Definition 4 (hyper-Wiener index). For a simple connected graph $G$, the hyper-Wiener index is denoted by WW $(G)$ and is defined as

$$
\mathrm{WW}(G)=\frac{1}{2} \sum_{y \in V(G)} \sum_{z \in V(G)}\left(d(y, z)+d(y, z)^{2}\right) .
$$

The hyper-Wiener index (HWI) was introduced by Randić [38] and is used to forecast physical chemistry characteristics of organic compounds.

Definition 5 (modified hyper-Wiener index). Another variant of Wiener index is hyper-Wiener index (MHWI) which is denoted by $\mathrm{WW}_{\lambda}(G)$. For a simple connected graph $G$, the modified Wiener index is defined as

$$
\mathrm{WW}_{\lambda}(G)=\frac{1}{2} \sum_{y \in V(G)} \sum_{z \in V(G)}\left(d(y, z)^{\lambda}+d(y, z)^{2 \lambda}\right),
$$

where $\lambda$ is any positive integer.

Definition 6 (Harary polynomial). The Harary polynomial for a simple connected graph $G$ is denoted by $h(G)$ and is defined as

$$
h(G)=\sum_{y \in V(G)} \sum_{z \in V(G)} \frac{1}{d(y, z)} x^{d(y, z)} .
$$


Definition 7 (generalized Harary index). The generalized Harary index for a simple connected graph $G$ is denoted by $h_{t}(G)$ and is defined as

$$
h_{t}(G)=\sum_{y \in V(G)} \sum_{z \in V(G)} \frac{1}{d(y, z)+t},
$$

where $t=1,2,3,4, \ldots$

For detailed study on Harary polynomial and Harary index, we refer to the readers $[39,40]$ and references therein.

Definition 8 (multiplicative Wiener index). The multiplicative Wiener index for a simple connected graph $G$ is denoted by $\pi(G)$ and is defined as

$$
\pi(G)=\prod_{\{y, z\} \subseteq V(G)} d(y, z) .
$$

\section{Methodology}

With the aid of distance matrix of a graph $G$, we can evaluate Hosoya polynomial. To calculate the Hosoya polynomial, we must calculate the number of pairs of vertices at distance $1,2,3, \ldots, \operatorname{dia}(G), \quad$ where $\operatorname{dia}(G)=\max \{d(u, v) ; u, v \in$ $V(G)\}$. Mathematical induction will be used for the above cause. The usual vision of Hosoya polynomial is given below, where $d$ represents the maximum distance in graph.

$$
H(G ; x)=a_{0}(n) x^{0}+a_{1}(n) x^{1}+a_{2}(n) x^{2}+\cdots+a_{d}(n) x^{d} .
$$

\section{Distance-Based Polynomials and Indices for Hierarchical Hypercube Networks}

This section consists of two subsections. In Section 4.1, we present results about HHC-1, and in Section 4.2, we present results about HHC-2.

4.1. Distance-Based Polynomials and Indices for Hierarchical Hypercube Network HHC-1. In Theorem 1, we give Hosoya polynomial of $\mathrm{HHC}-1$.

Theorem 1. For $n \geq 3$, the Hosoya polynomial of HHC -1 is

$$
\begin{aligned}
H(H H C-1 ; x)= & (20+24 n) x+(24+40 n) x^{2}+(24+64 n) x^{3}+(4+100 n) x^{4}+(-28+124 n) x^{5} \\
& +(-80+132 n) x^{6}+(-152+132 n) x^{7}+\sum_{8 \leq m \leq 2 n+2}((8(37-8 m+16 n))) x^{m}+108 x^{2 n+3}+60 x^{2 n+4}+20 x^{2 n+5}
\end{aligned}
$$

Proof. To prove this result, we need to calculate $\left|a_{m}(n)\right|=$ number of pair of vertices at distance $m$, where $m=1,2,3, \ldots, 2 n+5$. Using Figure 1, the number of pair of vertices at different distances is computed and is listed in Tables 1 and 2 .

Now, by using Table 1, we have

$$
\begin{aligned}
& \left|a_{1}(n)\right|=20+24 n, \\
& \left|a_{2}(n)\right|=24+40 n, \\
& \left|a_{3}(n)\right|=24+64 n, \\
& \left|a_{4}(n)\right|=4+100 n, \\
& \left|a_{5}(n)\right|=-28+124 n, \\
& \left|a_{6}(n)\right|=-80+132 n, \\
& \left|a_{7}(n)\right|=-152+132 n .
\end{aligned}
$$

The remaining proof is divided into the following two main cases.

Case 1 . When $m \equiv 0(\bmod 2)$ and $8<m \leq 2 n+2$.

It can be observed from Table 2 that

$$
\begin{aligned}
& \left|a_{8}(3)\right|=168, \\
& \left|a_{8}(4)\right|=296, \\
& \left|a_{8}(5)\right|=424, \\
& \left|a_{8}(6)\right|=552, \\
& \left|a_{8}(7)\right|=680, \\
& \left|a_{8}(8)\right|=808 .
\end{aligned}
$$

Now, we can deduce that

$$
\left|a_{8}(n)\right|=40+128(n-2) .
$$

In a similar fashion, we have

$$
\begin{aligned}
& \left|a_{10}(4)\right|=168, \\
& \left|a_{10}(5)\right|=296, \\
& \left|a_{10}(6)\right|=424, \\
& \left|a_{10}(7)\right|=552, \\
& \left|a_{10}(8)\right|=680, \\
& \left|a_{10}(9)\right|=808 .
\end{aligned}
$$


TABLE 1: Pair of vertices in HHC-1 at different distances.

\begin{tabular}{|c|c|c|c|c|c|c|c|c|c|}
\hline \multirow{2}{*}{$m$} & \multicolumn{9}{|c|}{$n$} \\
\hline & 3 & 4 & 5 & 6 & 7 & 8 & . & . & \\
\hline 1 & 92 & 116 & 140 & 164 & 188 & 212 & . & . & . \\
\hline 2 & 144 & 184 & 224 & 264 & 304 & 344 & . & . & 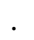 \\
\hline 3 & 216 & 280 & 344 & 408 & 472 & 536 & . & . & 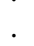 \\
\hline 4 & 304 & 404 & 504 & 604 & 704 & 804 & . & . & . \\
\hline 5 & 344 & 468 & 592 & 716 & 840 & 964 & . & . & . \\
\hline 6 & 316 & 448 & 580 & 712 & 844 & 976 & . & . & . \\
\hline 7 & 244 & 376 & 508 & 640 & 772 & 904 & . & . & . \\
\hline
\end{tabular}

TABle 2: Pair of vertices in HHC-1 at different distances.

\begin{tabular}{|c|c|c|c|c|c|c|c|c|c|}
\hline \multirow{2}{*}{$m$} & \multicolumn{8}{|c|}{$n$} & \\
\hline & 3 & 4 & 5 & 6 & 7 & 8 & . & . & . \\
\hline 8 & 168 & 296 & 424 & 552 & 680 & 808 & . & . & . \\
\hline 9 & - & 232 & 360 & 488 & 616 & 744 & . & . & . \\
\hline 10 & - & 168 & 296 & 424 & 552 & 680 & . & . & . \\
\hline 11 & - & - & 232 & 360 & 488 & 616 & . & . & . \\
\hline 12 & - & - & 168 & 296 & 424 & 552 & . & . & . \\
\hline 13 & - & - & - & 232 & 360 & 488 & . & . & . \\
\hline 14 & - & - & - & 168 & 296 & 424 & . & . & . \\
\hline 15 & - & - & - & - & 232 & 360 & . & . & . \\
\hline 16 & - & - & - & - & 168 & 296 & . & . & . \\
\hline 17 & - & - & - & - & - & 232 & . & . & . \\
\hline 18 & - & - & - & - & - & 168 & . & . & . \\
\hline 19 & - & - & - & - & - & - & . & . & . \\
\hline 20 & - & - & - & - & - & - & - & . & . \\
\hline 21 & - & - & - & - & - & - & - & - & \\
\hline
\end{tabular}

It implies that

$$
\left|a_{10}(n)\right|=40+128(n-3) .
$$

In a similar fashion, we infer

$$
\begin{aligned}
& \left|a_{12}(n)\right|=40+128(n-4), \\
& \left|a_{14}(n)\right|=40+128(n-5), \\
& \left|a_{16}(n)\right|=40+128(n-6),
\end{aligned}
$$

Now, we have

$$
\left|a_{m}(n)\right|=40+128\left(n-\frac{(m-4)}{2}\right)=8(37-8 m+16 n) \text {. }
$$

Case 2. When $m \equiv 1(\bmod 2)$ and $9<m \leq 2 n+1$.

It can be observed from Table 2 that

$$
\begin{aligned}
& \left|a_{9}(4)\right|=232 \\
& \left|a_{9}(5)\right|=360 \\
& \left|a_{9}(6)\right|=488 \\
& \left|a_{9}(7)\right|=616 \\
& \left|a_{9}(8)\right|=744 \\
& \left|a_{9}(9)\right|=872
\end{aligned}
$$

Now, we can deduce that

$$
\left|a_{9}(n)\right|=104+128(n-3) \text {. }
$$

By means of the same trick, we obtain

$$
\begin{aligned}
\left|a_{11}(5)\right| & =232 \\
\left|a_{11}(6)\right| & =360 \\
\left|a_{11}(7)\right| & =488 \\
\left|a_{11}(8)\right| & =616 \\
\left|a_{11}(9)\right| & =744 \\
\left|a_{11}(10)\right| & =872
\end{aligned}
$$

which reveal that

$$
\left|a_{11}(n)\right|=104+128(n-4) .
$$

With a similar approach, we get

$$
\begin{aligned}
& \left|a_{13}(n)\right|=104+128(n-5), \\
& \left|a_{15}(n)\right|=104+128(n-6), \\
& \left|a_{17}(n)\right|=104+128(n-7),
\end{aligned}
$$

Hence, we have

$$
\begin{aligned}
\left|a_{m}(n)\right| & =104+128\left(n-\frac{(m-3)}{2}\right)\left|a_{m}(n)\right| \\
& =8(37-8 m+16 n) .
\end{aligned}
$$


One can see that in both cases, we get the same result, so we can write for $8<m \leq 2 n+1$ as

$$
\left|a_{m}(n)\right|=8(37-8 m+16 n),
$$

and the remaining last three distances having fixed values are

$$
\begin{aligned}
& \left|a_{2 n+3}(n)\right|=108 \\
& \left|a_{2 n+4}(n)\right|=60 \\
& \left|a_{2 n+5}(n)\right|=20 .
\end{aligned}
$$

By what have been mentioned above and using definition of Hosoya polynomial, we arrive at our desired result.

In Theorem 2, we give Harary polynomial for HHC-1.

$$
\begin{aligned}
h(\mathrm{HHC}-1 ; x)= & (20+24 n) x+\frac{(24+40 n)}{2} x^{2}+\frac{(24+64 n)}{3} x^{3}+\frac{(4+100 n)}{4} x^{4}+\frac{(-28+124 n)}{5} x^{5} \\
& +\frac{(-80+132 n)}{6} x^{6}+\frac{(-152+132 n)}{7} x^{7} \\
& +\sum_{8 \leq m \leq 2 n+2} \frac{(8(37-8 m+16 n))}{m} x^{m}+\frac{108}{2 n+3} x^{2 n+3}+\frac{60}{2 n+4} x^{2 n+4}+\frac{20}{2 n+5} x^{2 n+5}
\end{aligned}
$$

Proof. The proof of this result is easy to follow by using information given in Theorem 1 and definition of Harary polynomial.

In Theorem 3, we give modified Wiener index, modified hyper-Wiener index, generalized Harary index, and multiplicative Wiener index of HHC-1.
Theorem 3. For $H H C-1$, we have

(1) The modified Wiener index:

$$
\begin{aligned}
W_{\lambda}(\mathrm{HHC}-1)= & (20+24 n) 1^{\lambda}+(24+40 n) 2^{\lambda}+(24+64 n) 3^{\lambda}+(4+100 n) 4^{\lambda}+(-28+124 n) 5^{\lambda} \\
& +(-80+132 n) 6^{\lambda}+(-152+132 n) 7^{\lambda} \\
& +\sum_{8 \leq m \leq 2 n+2}(8(37-8 m+16 n))\left(m^{\lambda}\right)+(108)(2 n+3)^{\lambda}+(60)(2 n+4)^{\lambda}+(20)(2 n+5)^{\lambda}
\end{aligned}
$$

(2) The modified hyper-Wiener index:

$$
\begin{aligned}
\mathrm{WW}_{\lambda}(\mathrm{HHC}-1)= & (20+24 n)\left(1^{\lambda}+1^{2 \lambda}\right) \\
& +(24+40 n)\left(2^{\lambda}+2^{2 \lambda}\right)+(24+64 n)\left(3^{\lambda}+3^{2 \lambda}\right) \\
& +(4+100 n)\left(4^{\lambda}+4^{2 \lambda}\right)+(-28+124 n)\left(5^{\lambda}+5^{2 \lambda}\right)+(-80+132 n)\left(6^{\lambda}+6^{2 \lambda}\right)+(-152+132 n)\left(7^{\lambda}+7^{2 \lambda}\right) \\
& +\sum_{8 \leq m \leq 2 n+2}(8(37-8 m+16 n))\left(m^{\lambda}+m^{2 \lambda}\right)+(108)\left((2 n+3)^{\lambda}+(2 n+3)^{2 \lambda}\right) \\
& +(60)\left((2 n+4)^{\lambda}+(2 n+4)^{2 \lambda}\right)+(20)\left((2 n+5)^{\lambda}+(2 n+5)^{2 \lambda}\right) .
\end{aligned}
$$


(3) The generalized Harary index:

$$
\begin{aligned}
h_{t}(\mathrm{HHC}-1)= & \frac{(20+24 n)}{1+t}+\frac{(24+40 n)}{2+t}+\frac{(24+64 n)}{3+t}+\frac{(4+100 n)}{4+t}+\frac{(-28+124 n)}{5+t} \\
& +\frac{(-80+132 n)}{6+t}+\frac{(-152+132 n)}{7+t} \\
& +\sum_{8 \leq m \leq 2 n+2}(8(37-8 m+16 n)) \frac{1}{m+t}+\frac{108}{2 n+3+t}+\frac{60}{2 n+4+t}+\frac{20}{2 n+5+t} .
\end{aligned}
$$

(4) The multiplicative Wiener index:

$$
\begin{aligned}
\pi(\mathrm{HHC}-1)= & 1^{(20+24 n)} \times 2^{(24+40 n)} \times 3^{(24+64 n)} \times 4^{(4+100 n)} \times 5^{(-28+124 n)} \times 6^{(-80+132 n)} \times 7^{(-152+132 n)} \\
& \times \prod_{8<m \leq 2 n+2} m^{(8(37-8 m+16 n))} \times(2 n+3)^{108} \times(2 n+4)^{60} \times(2 n+5)^{20} .
\end{aligned}
$$

From Theorem 3, we get the following results immediately.

Corollary 1. For $H H C-1$, we have

$$
W(\mathrm{HHC}-1)=\frac{256 n^{3}}{3}+592 n^{2}+\frac{2696 n}{3}+376 .
$$

Proof. This result can be easily established by taking $\lambda=1$ in (2) of Theorem 3.

Proof. This result can be easily established by taking $\lambda=1$ in (1) of Theorem 3.

$$
\begin{aligned}
W W(H H C-1)= & \frac{256 n^{4}}{3}+\frac{2624 n^{3}}{3}+\frac{10544 n^{2}}{3} \\
& +\frac{14056 n}{3}+1816
\end{aligned}
$$

Corollary 2. For $H H C-1$, we have

Corollary 3. For $H H C-1$, we have

$$
\begin{aligned}
H(\mathrm{HHC}-1)= & \frac{15599 n}{100}+\frac{6347931761169771}{18014398509481984} \\
& +\sum_{8 \leq m \leq 2 n+2} \frac{(8(37-8 m+16 n))}{m}+\frac{108}{2 n+3}+\frac{20}{2 n+5}+\frac{30}{n+2}
\end{aligned}
$$


Proof. This result can be easily established by taking $t=1$ in (3) of Theorem 3.
4.2. Distance-Based Polynomials and Indices for Hierarchical Hypercube Network HHC-2. In Theorem 4, we give Hosoya polynomial for HHC-2.

Theorem 4. For HHC-2, the Hosoya polynomial is

$$
\begin{aligned}
H(H H C-2 ; x)= & (28+32 n) x+(24+48 n) x^{2}+(36+96 n) x^{3}+(-4+124 n) x^{4}+(-60+128 n) x^{5} \\
& +(-120+132 n) x^{6}+\sum_{m=0(\bmod 2), 8 \leq m \leq 2 n+2}(8(33-8 m+16 n)) x^{m} \\
& +\sum_{m \equiv 1(\bmod 2), 7 \leq m \leq 2 n+1}(-64(m-2(2+n))) x^{m}+68 x^{2 n+3}+32 x^{2 n+4}+4 x^{2 n+5}
\end{aligned}
$$

Proof. To prove this result, we have to calculate $\left|a_{m}(n)\right|$ where $m=1,2,3, \ldots, 2 n+5$. Here $\left|a_{m}(n)\right|$ is the same as in Theorem 1. From Figure 2, we can compute the number of pair of vertices in HHC-2 at different distances, which is given in Tables 3 and 4 .

Now from Table 3, we have

$$
\begin{aligned}
& \left|a_{1}(n)\right|=28+32 n, \\
& \left|a_{2}(n)\right|=24+48 n, \\
& \left|a_{3}(n)\right|=36+96 n, \\
& \left|a_{4}(n)\right|=-4+124 n \\
& \left|a_{5}(n)\right|=-60+128 n, \\
& \left|a_{6}(n)\right|=-120+132 n
\end{aligned}
$$

The remaining proof is divided into the following two main cases.

Case 1 . When $m \equiv 0(\bmod 2)$ and $8<m \leq 2 n+2$.

It can be observed from Table 4 that

$$
\begin{aligned}
& \left|a_{8}(3)\right|=136, \\
& \left|a_{8}(4)\right|=264, \\
& \left|a_{8}(5)\right|=392, \\
& \left|a_{8}(6)\right|=520 \\
& \left|a_{8}(7)\right|=648 \\
& \left|a_{8}(8)\right|=776 .
\end{aligned}
$$

Now, we can deduce that

$$
\left|a_{8}(n)\right|=8+128(n-2) .
$$

In a similar fashion, we have

$$
\begin{aligned}
& \left|a_{10}(4)\right|=136, \\
& \left|a_{10}(5)\right|=264, \\
& \left|a_{10}(6)\right|=392, \\
& \left|a_{10}(7)\right|=520, \\
& \left|a_{10}(8)\right|=648, \\
& \left|a_{10}(9)\right|=776 .
\end{aligned}
$$

It implies that

$$
\left|a_{10}(n)\right|=8+128(n-3) .
$$

In a similar fashion, we infer

$$
\begin{aligned}
\left|a_{12}(n)\right| & =8+128(n-4), \\
\left|a_{14}(n)\right| & =8+128(n-5) \\
\left|a_{16}(n)\right| & =8+128(n-6) \\
\cdots &
\end{aligned}
$$

which yield

$$
\left|a_{m}(n)\right|=8+128\left(n-\frac{(m-4)}{2}\right)=8(33-8 m+16 n) \text {. }
$$

Case 2. When $m \equiv 1(\bmod 2)$ and $7<m \leq 2 n+1$.

It can be observed from Table 4 that

$$
\begin{aligned}
& \left|a_{7}(3)\right|=192, \\
& \left|a_{7}(4)\right|=320, \\
& \left|a_{7}(5)\right|=448, \\
& \left|a_{7}(6)\right|=576, \\
& \left|a_{7}(7)\right|=704, \\
& \left|a_{7}(8)\right|=832 .
\end{aligned}
$$

Now, we can deduce that 
TABLE 3: Number of pair of vertices in HHC-2 at different distances.

\begin{tabular}{|c|c|c|c|c|c|c|c|c|c|}
\hline \multirow{2}{*}{$m$} & \multicolumn{8}{|c|}{$n$} & \\
\hline & 2 & 3 & 4 & 5 & 6 & 7 & . & . & . \\
\hline 1 & 92 & 124 & 156 & 188 & 220 & 252 & . & . & - \\
\hline 2 & 120 & 168 & 216 & 264 & 312 & 360 & . & . & . \\
\hline 3 & 228 & 324 & 420 & 516 & 612 & 708 & . & . & . \\
\hline 4 & 244 & 368 & 492 & 616 & 740 & 864 & . & . & . \\
\hline 5 & 196 & 324 & 452 & 580 & 708 & 836 & . & . & . \\
\hline 6 & 144 & 276 & 408 & 540 & 672 & 804 & . & . & . \\
\hline
\end{tabular}

TABLE 4: Number of pair of vertices of HHC-2 at different distances.

\begin{tabular}{|c|c|c|c|c|c|c|c|c|c|}
\hline \multirow{2}{*}{$m$} & \multicolumn{8}{|c|}{$n$} & \\
\hline & 3 & 4 & 5 & 6 & 7 & 8 & . & . & \\
\hline 7 & 192 & 320 & 448 & 576 & 704 & 832 & . & . & . \\
\hline 8 & 136 & 264 & 392 & 520 & 648 & 776 & . & . & \\
\hline 9 & - & 192 & 320 & 448 & 576 & 704 & . & . & . \\
\hline 10 & - & 136 & 264 & 392 & 520 & 648 & . & . & \\
\hline 11 & - & - & 192 & 320 & 448 & 576 & . & . & . \\
\hline 12 & - & - & 136 & 264 & 392 & 520 & . & . & . \\
\hline 13 & - & - & - & 192 & 320 & 448 & . & . & . \\
\hline 14 & - & - & - & 136 & 264 & 392 & . & . & . \\
\hline 15 & - & - & - & - & 192 & 320 & . & . & . \\
\hline 16 & - & - & - & - & 136 & 264 & . & . & . \\
\hline 17 & - & - & - & - & - & 192 & . & . & . \\
\hline 18 & - & - & - & - & - & 136 & . & . & . \\
\hline 19 & - & - & - & - & - & - & . & . & . \\
\hline 20 & - & - & - & - & - & - & - & . & . \\
\hline 21 & - & - & - & - & - & - & - & - & \\
\hline
\end{tabular}

$$
\left|a_{7}(n)\right|=64+128(n-2) .
$$

So, we obtain

$$
\begin{aligned}
& \left|a_{9}(4)\right|=192, \\
& \left|a_{9}(5)\right|=320, \\
& \left|a_{9}(6)\right|=448, \\
& \left|a_{9}(7)\right|=576, \\
& \left|a_{9}(8)\right|=704, \\
& \left|a_{9}(9)\right|=832,
\end{aligned}
$$

which reveal that

$$
\left|a_{9}(n)\right|=64+128(n-3) .
$$

Also, we get

$$
\begin{aligned}
& \left|a_{13}(n)\right|=64+128(n-4), \\
& \left|a_{15}(n)\right|=64+128(n-5), \\
& \left|a_{17}(n)\right|=64+128(n-6),
\end{aligned}
$$

Hence, we have

$$
\begin{aligned}
\left|a_{m}(n)\right| & =64+128\left(n-\frac{(m-3)}{2}\right) \\
& =-64(m-2(2+n)),
\end{aligned}
$$

and the remaining last three distances having fixed values are

$$
\begin{aligned}
& \left|a_{2 n+3}(n)\right|=68, \\
& \left|a_{2 n+4}(n)\right|=32, \\
& \left|a_{2 n+5}(n)\right|=4 .
\end{aligned}
$$

By what have been mentioned above and using the definition of Hosoya polynomial, we arrive at our desired result.

In Theorem 5, we give Harary polynomial for HHC-2.

Theorem 5. For HHC-2, the Harary polynomial is 


$$
\begin{aligned}
h(\mathrm{HHC}-2 ; x)= & (28+32 n) x+\frac{(24+48 n)}{2} x^{2}+\frac{(36+96 n)}{3} x^{3}+\frac{(36+96 n)}{4} x^{4}+\frac{(-60+128 n)}{5} x^{5} \\
& +\frac{(-120+132 n)}{6} x^{6}+\sum_{8 \leq m \leq 2 n+2} \frac{(8(33-8 m+16 n))}{m} x^{m} \\
& +\sum_{7 \leq m \leq 2 n+1} \frac{(-64(m-2(2+n)))}{m} x^{m}+\frac{68}{2 n+3} x^{2 n+3}+\frac{32}{2 n+4} x^{2 n+4}+\frac{4}{2 n+5} x^{2 n+5} .
\end{aligned}
$$

Proof. The proof of this theorem is straightforward from the facts specified in Theorem 4 and by definition of Harary polynomial.

In Theorem 6, we give modified Wiener index, modified hyper-Wiener index, generalized Harary index, and multiplicative Wiener index for HHC-2.
Theorem 6. For $\mathrm{HHC}-2$, we have

(1) The modified Wiener index:

$$
\begin{aligned}
W_{\lambda}(\mathrm{HHC}-2)= & (28+32 n) 1^{\lambda}+(24+48 n) 2^{\lambda}+(36+96 n) 3^{\lambda}+(36+96 n) 4^{\lambda}+(-60+128 n) 5^{\lambda} \\
& +(-120+132 n) 6^{\lambda}+\sum_{m \equiv 0(\bmod 2), 8 \leq m \leq 2 n+2}(8(33-8 m+16 n))\left(m^{\lambda}\right) \\
& +\sum_{m \equiv 1(\bmod 2), 7 \leq m \leq 2 n+1}(-64(m-2(2+n)))\left(m^{\lambda}\right)+(68)(2 n+3)^{\lambda}+(32)(2 n+4)^{\lambda}+(4)(2 n+5)^{\lambda} .
\end{aligned}
$$

(2) The modified hyper-Wiener index:

$$
\begin{aligned}
\mathrm{WW}_{\lambda}(\mathrm{HHC}-2)= & (28+32 n)\left(1^{\lambda}+1^{2 \lambda}\right)+(24+48 n)\left(2^{\lambda}+2^{2 \lambda}\right)+(36+96 n)\left(3^{\lambda}+3^{2 \lambda}\right) \\
& +(36+96 n)\left(4^{\lambda}+4^{2 \lambda}\right)+(-60+128 n)\left(5^{\lambda}+5^{2 \lambda}\right)+(-120+132 n)\left(6^{\lambda}+6^{2 \lambda}\right) \\
& +\sum_{m=0(\bmod 2), 8 \leq m \leq n+2}(8(33-8 m+16 n))\left(m^{\lambda}+m^{2 \lambda}\right) \\
& +\sum_{m=1(\bmod 2), 7 \leq m \leq n+1}(-64(m-2(2+n)))\left(m^{\lambda}+m^{2 \lambda}\right) \\
& \quad \sum^{2 \lambda}\left((2 n+3)^{\lambda}+(2 n+3)^{2 \lambda}\right)+(32)\left((2 n+4)^{\lambda}+(2 n+4)^{2 \lambda}\right)+(4)\left((2 n+5)^{\lambda}+(2 n+5)^{2 \lambda}\right)
\end{aligned} .
$$

(3) The generalized Harary index:

$$
\begin{aligned}
h_{t}(\mathrm{HHC}-2)= & \frac{(28+32 n)}{1+t}+\frac{(24+48 n)}{2+t}+\frac{(36+96 n)}{3+t}+\frac{(36+96 n)}{4+t}+\frac{(-60+128 n)}{5+t}+\frac{(-120+132 n)}{6+t} \\
& +\sum_{m \equiv 0(\bmod 2), 8 \leq m \leq 2 n+2}(8(33-8 m+16 n)) \frac{1}{m+t} \\
& +\sum_{m \equiv 1(\bmod 2), 7 \leq m \leq 2 n+1}(-64(m-2(2+n))) \frac{1}{m+t}+\frac{108}{2 n+3+t}+\frac{60}{2 n+4+t}+\frac{20}{2 n+5+t} .
\end{aligned}
$$


(4) The multiplicative Wiener index:

$$
\begin{aligned}
\pi(\mathrm{HHC}-2)= & 1^{(28+32 n)} \times 2^{(24+48 n)} \times 3^{(36+96 n)} \times 4^{(36+96 n)} \times 5^{(-60+128 n)} \times 6^{(-120+132 n)} \\
& \times \prod_{m=0(\bmod 2), 8<m \leq 2 n+2} m^{(8(33-8 m+16 n))} \times \prod_{m \equiv 1(\bmod 2), 7<m \leq 2 n+1} m^{(-64(m-2(2+n)))}(2 n+3)^{68} \\
& \times(2 n+4)^{32} \times(2 n+5)^{4} .
\end{aligned}
$$

From Theorem 6, we get the following results immediately.

Corollary 4. For $\mathrm{HHC}-2$, we have

$$
\begin{aligned}
\mathrm{WW}(\mathrm{HHC}-2)= & \frac{256 n^{4}}{3}+\frac{2336 n^{3}}{3}+\frac{8264 n^{2}}{3} \\
& +\frac{10480 n}{3}+1320 .
\end{aligned}
$$

$$
W(\mathrm{HHC}-2)=\frac{256 n^{3}}{3}+520 n^{2}+\frac{2288 n}{3}+316 .
$$

Proof. This result can be easily established by taking $\lambda=1$ in (1) of Theorem 6.

Proof. This result can be easily established by taking $\lambda=1$ in (2) of Theorem 6.

Corollary 6. For $H H C-2$, we have

Corollary 5. For $\mathrm{HHC}-2$, we have

$$
\begin{aligned}
H(\mathrm{HHC}-2)= & \frac{798 n}{5}+29+\sum_{m \equiv 0(\bmod 2), 8 \leq m \leq 2 n+2}(8(33-8 m+16 n)) \frac{1}{m} \\
& +\sum_{m \equiv 1(\bmod 2), 7 \leq m \leq 2 n+1}(-64(m-2(2+n))) \frac{1}{m}+\frac{108}{2 n+3}+\frac{20}{2 n+5}+\frac{30}{n+2} .
\end{aligned}
$$

Proof. This result can be easily established by taking $t=1$ in (3) of Theorem 6.

\section{Conclusion}

Topological indices can be applied in different fields of science, such as material science, arithmetic, informatics, biology, and so on. However, the most critical use of topological indices to date is in the nonexact quantitative structure-property relationships and quantitative structureactivity relationships. In this paper, we studied hierarchical hypercube networks. We computed distance-based polynomials and distance-based indices for these networks. In fact, we computed Hosoya polynomials, Harary polynomials, Wiener index, and different variants of Wiener indices for the studied networks. Our results can help in understanding the topology of hierarchical hypercube networks and can be used to solve integral equations.

\section{Data Availability}

The data used to support the findings of this study are included within the article.

\section{Conflicts of Interest}

The authors declare that they have no conflicts of interest.

\section{Authors' Contributions}

Tingmei Gao wrote the final version of this paper, analyzed the results, and arranged funding for this study. Iftikhar Ahmed proved the results and wrote the first version of this paper.

\section{References}

[1] S. C. Basak, D. Mills, B. D. Gute, G. D. Grunwald, and A. T. Balaban, "Applications of topological indices in the property/bioactivity/toxicity prediction of chemicals," in Topology in Chemistry, pp. 113-184, Woodhead Publishing, Sawston, UK, 2002.

[2] M. P. Hanson and D. H. Rouvray, "Novel applications of topological indices. 2. Prediction of the threshold soot index for hydrocarbon fuels," The Journal of Physical Chemistry, vol. 91, no. 11, pp. 2981-2985, 1987.

[3] P. J. Hansen and P. C. Jurs, "Chemical applications of graph theory. Part I. Fundamentals and topological indices," Journal of Chemical Education, vol. 65, no. 7, p. 574, 1988. 
[4] W. Gao, W. Wang, and M. R. Farahani, "Topological indices study of molecular structure in anticancer drugs," Journal of Chemistry, vol. 2016, Article ID 3216327, 8 pages, 2016.

[5] S. Hayat and M. Imran, "Computation of topological indices of certain networks," Applied Mathematics and Computation, vol. 240, no. 23, pp. 213-228, 2014.

[6] S. Hayat and M. Imran, "Computation of certain topological indices of nanotubes covered by C5 and C7," Journal of Computational and Theoretical Nanoscience, vol. 12, no. 4, pp. 533-541, 2015.

[7] A. Q. Baig, M. Imran, and H. Ali, "On topological indices of poly oxide, poly silicate, DOX, and DSL networks," Canadian Journal of Chemistry, vol. 93, no. 7, pp. 730-739, 2015.

[8] Y. Bashir, A. Aslam, M. Kamran et al., "On forgotten topological indices of some dendrimers structure," Molecules, vol. 22 , no. 6 , p. $867,2017$.

[9] A. Aslam, J. L. G. Guirao, S. Ahmad, and W. Gao, "Topological indices of the line graph of subdivision graph of complete bipartite graphs," Applied Mathematics \& Information Sciences, vol. 11, no. 6, pp. 1631-1636, 2017.

[10] G. C. Marsden, P. J. Marchand, P. Harvey, and S. C. Esener, "Optical transpose interconnection system architectures," Optics letters, vol. 18, no. 13, pp. 1083-1085, 1993.

[11] A. Al-Ayyoub, A. Awwad, K. Day, and M. Ould-Khaoua, "Generalized methods for algorithm development on optical systems," The Journal of Supercomputing, vol. 38, no. 2, pp. 111-125, 2006.

[12] C. F. Wang and S. Sahni, "Matrix multiplication on the OTISmesh optoelectronic computer," IEEE Transactions on Computers, vol. 50, no. 7, pp. 635-646, 2001.

[13] C. F. Wang and S. Sahni, "Image processing on the OTISmesh optoelectronic computer," IEEE Transactions on Parallel and Distributed Systems, vol. 11, no. 2, pp. 97-109, 2000.

[14] C. F. Wang and S. Sahni, "Basic operations on the OTIS-mesh optoelectronic computer," IEEE Transactions on Parallel and Distributed Systems, vol. 9, no. 12, pp. 1226-1236, 1998.

[15] S. Rajasekaran and S. Sahni, "Randomized routing, selection, and sorting on the OTIS-mesh," IEEE Transactions on Parallel and Distributed Systems, vol. 9, no. 9, pp. 833-840, 1998.

[16] H. Hosoya, "On some counting polynomials in chemistry," Discrete Applied Mathematics, vol. 19, no. 1-3, pp. 239-257, 1988.

[17] D. Stevanović, "Hosoya polynomial of composite graphs," Discrete Mathematics, vol. 235, no. 1-3, pp. 237-244, 2001.

[18] M. R. Farahani, "On the Schultz polynomial and Hosoya polynomial of circumcoronene series of benzenoid," Journal of Applied Mathematics \& Informatics, vol. 31, no. 5-6, pp. 595-608, 2013.

[19] J. M. Kumar and L. M. Patnaik, "Extended hypercube: a hierarchical interconnection network of hypercubes," IEEE Transactions on Parallel and Distributed Systems, vol. 3, no. 1, pp. 45-57, 1992.

[20] R.-Y. Wu, G.-H. Chen, Y.-L. Kuo, and G. J. Chang, "Nodedisjoint paths in hierarchical hypercube networks," Information Sciences, vol. 177, no. 19, pp. 4200-4207, 2007.

[21] Q. M. Malluhi and M. A. Bayoumi, "The hierarchical hypercube: a new interconnection topology for massively parallel systems," IEEE Transactions on Parallel and Distributed Systems, vol. 5, no. 1, pp. 17-30, 1994.

[22] S. K. Yun and K. H. Park, "Comments on "Hierarchical cubic networks", IEEE Transactions on Parallel and Distributed Systems vol. 9, no. 4, pp. 410-414, 1998.

[23] M. Abd-El-Barr and T. F. Al-Somani, "Performance comparison of hierarchical interconnection networks," in
Proceedings of 2011 IEEE Pacific Rim Conference on Communications, Computers and Signal Processing, pp. 191-196, IEEE, Victoria, British Columbia (BC), Canada, August 2011.

[24] J.-S. Fu and G.-H. Chen, "Hamiltonicity of the hierarchical cubic network," Theory of Computing Systems, vol. 35, no. 1, pp. 59-79, 2002.

[25] C. Chen and D. P. Agrawal, "dBCube: a new class of hierarchical multiprocessor interconnection networks with area efficient layout," IEEE Transactions on Parallel and Distributed Systems, vol. 4, no. 12, pp. 1332-1344, 1993.

[26] S.-K. Yun and K. H. Park, "Hierarchical hypercube networks (HHN) for massively parallel computers," Journal of Parallel and Distributed Computing, vol. 37, no. 2, pp. 194-199, 1996.

[27] S. P. Dandamudi and D. L. Eager, "On hypercube-based hierarchical interconnection network design," Journal of Parallel and Distributed Computing, vol. 12, no. 3, pp. 283289, 1991.

[28] C. H. Yeh and B. Parhami, "Swapped networks: unifying the architectures and algorithms of a wide class of hierarchical parallel processors," in Proceedings of 1996 International Conference on Parallel and Distributed Systems, pp. 230-237, IEEE, Tokyo, Japan, June 1996.

[29] K. Ghose and K. R. Desai, "Hierarchical cubic networks," IEEE Transactions on Parallel and Distributed Systems, vol. 6, no. 4, pp. 427-435, 1995.

[30] M. B. Galles and D. E. Lenoski, U.S. Patent No. 5,669,008, U.S. Patent and Trademark Office, Washington, USA, 1997.

[31] C. H. Yeh and B. Parhami, "Hierarchical swapped networks: efficient low-degree alternatives to hypercubes and generalized hypercubes," in Proceedings Second International Symposium on Parallel Architectures, Algorithms, and Networks (ISPAN'96), pp. 90-96, IEEE, Beijing, China, June 1996.

[32] W.-K. Chiang and R.-J. Chen, "Topological properties of hierarchical cubic networks," Journal of Systems Architecture, vol. 42, no. 4, pp. 289-307, 1996.

[33] M. Abd-El-Barr and T. F. Al-Somani, "Topological properties of hierarchical interconnection networks: a review and comparison," Journal of Electrical and Computer Engineering, vol. 2011, Article ID 189434, 12 pages, 2011.

[34] S. P. Dandamudi and D. L. Eager, "Hierarchical interconnection networks for multicomputer systems," IEEE Transactions on Computers, vol. 39, no. 6, pp. 786-797, 1990.

[35] M. Numan, S. I. Butt, S. I. Butt, and A. Taimur, "Super cyclic antimagic covering for some families of graphs," Open Journal of Mathematical Sciences, vol. 5, no. 1, pp. 27-33, 2021.

[36] H. M. Nagesh and V. R. Girish, "On the entire Zagreb indices of the line graph and line cut-vertex graph of the subdivision graph," Open Journal of Mathematical Sciences, vol. 4, no. 1, pp. $470-475,2020$.

[37] H. Wiener, "Structural determination of paraffin boiling points," Journal of the American Chemical Society, vol. 69, no. 1, pp. 17-20, 1947.

[38] M. Randic, "Index," Oral Cancer, vol. 49, no. 3, pp. 483-496, 2002.

[39] B. Lucic, A. Milicevic, S. Nikolic, and N. Trinajstic, "Harary index-twelve years later," Croatica Chemica Acta, vol. 75, no. 4, pp. 847-868, 2002.

[40] B. Zhou, X. Cai, and N. Trinajstić, “On Harary index," Journal of Mathematical Chemistry, vol. 44, no. 2, pp. 611-618, 2008. 